\title{
The Observational Research Study with the Trends in Healthcare Training and Leadership Ethics in The United States
}

http://doi.org/10.21272/bel.4(3).6-24.2020

Terrence D. Probst

Ed.D. MBA, MHR, COR II, Business Manager, Specialty Care Clinics, San Francisco VA Health Care System, USA

Karina Kasztelnik, ORCID: https://orcid.org/0000-0002-1090-3700

Ph.D., MBA, CPA, CTP, Professor of Accounting, Colorado State University, Global Campus, Aurora, CO, USA

\begin{abstract}
This paper summarizes how healthcare training has an influence on the leadership ethics in the U.S. This article includes four primary sections. The first section is the background to the problem, which provides a historical overview of the research on the problem being studied and justifies the need for this study. The second section provides the theoretical foundation models and theories which frame the variables and the research questions. The third section provides an in-depth discussion of various factors related to the problem statement, ultimately providing the population, the variables, the methodology, and the design. The fourth section synthesizes the prior three sections justifying the ten strategic points that frame the study. The methodology is the systematization literature review within this context and approaches for underling current trends in healthcare training in the U.S. Little is known regarding the association between ongoing healthcare refresher training and the severity of among direct patient care staff. This calls for the evaluation of leadership within the healthcare industry and the various strategies employed in finding out how to fund and implement ongoing healthcare refresher trainings for staff members. There is a scarcity of literature focusing on the characteristics of the implementation of healthcare protocols that impact the effectiveness of the programs. The results of the critical review article with analysis both authors can be useful for any business around the World to support and the improvement management decisions.
\end{abstract}

Keywords: Healthcare, Health Care Leadership, Ethics, Training, Management.

JEL Classification: I15, M53.

This work is licensed under a Creative Commons Attribution 4.0 International License

Cite as: Probst, D.T., Kasztelnik, K. (2020). The Observational Research Study with the Trends in Healthcare Training and Leadership Ethics in The United States. Business Ethics and Leadership, 4(3), 6-24. http://doi.org/10.21272/bel.4(3).6-24.2020.

(C) The Authors, 2020. This article is published with open access at Sumy State University.

\section{Introduction}

Healthcare workers (HCW's) or direct patient care staff play a critical role in the promotion of quality health services among homes and communities (Olson et al., 2016). Despite the demands of the job, direct patient care staff are still exposed to injuries and career-ending injuries because of the nature of their work (Bonomi et al., 2017; Olson et al., 2016; Van der Beek et al., 2017). Based on the 2016 Bureau of Labor Statistics, out of the 1,388 work-related injuries, 19 percent were from healthcare practitioners. Job-related injuries in the healthcare industry pose a serious threat to the overall implementation and service programs provided by healthcare institutions (Olson et al., 2016; Van der Beek et al., 2017).

Search Strategy. A comprehensive search of databases was done to ensure a thorough review of the relevant literature. Databases were accessed through a library database, and the general advanced search tool was used to search all databases. The researcher also employed the use of google scholar and examined the reference sections of obtained articles to help narrow the search. Search terms used included healthcare trainings, "Safe Patient Handling and Movement, " injury, healthcare, direct care, lift, lifting, low back pain, safety, work, work culture, safety training, and Ergonomics. As many combinations of the above terms as could be arranged were also used. Also, Google Scholar's "cited in" function was used to find other studies relevant to the topic. In addition to searching through databases, many other search strategies were used. A snowball research strategy 
involved identifying additional relevant articles from empirical research articles that have already been reviewed. Also, the researcher identified and reviewed monthly periodicals that focused on the research problem. The researcher attended healthcare training conventions to meet with various individuals in the field, which ultimately led to the identification of additional references.

Background of the Problem. The amount of injuries to healthcare staff has not gone unnoticed. States across the country have passed requirements that hospitals develop healthcare training protocols. These policy changes have resulted in improving the rate and quality of safety training for direct patient care staff (Lee et al., 2015). However, there is a broad variety of approaches that care facilities have taken to deal with the high injury rate among its staff. Some facilities have implemented healthcare training programs, which have been shown to reduce the number of injuries and workers' compensation claims, resulting in lower costs for the institution and better outcomes for patients (Vollman \& Bassett, 2014). However, it was found that the initial healthcare training were not sustained over time (Martin et al., 2009; Theis \& Finkelstein, 2014). It is well known and documented that direct patient care staff are at an increased risk for healthcare training and that the associated costs of the frequency of healthcare training are immense. This research aims to determine if increasing the frequency of ongoing healthcare training refresher training can reduce the rate of healthcare training over time. The next section of the review will discuss the various methods facilities have used to try to reduce the rate of injury among their staff and their varying degrees of success. Some possible explanations for inconsistent results could be the lack of the proper and functioning equipment, erratic use of healthcare training equipment among staff, insufficient training, lack of awareness, lack of leadership ethics buy-in regarding staff safety, and/or little to no healthcare training education before entering the profession (Olinski \& Norton, 2017).

The literature on job-related injuries also entails understanding the culture of the workplace and perceptions on workplace safety. There is ambiguity in the literature regarding what this means, but it does seem to have an impact on the outcomes of certain training programs. Perhaps the most perplexing part of the literature on healthcare training is the sheer volume of studies which document the frequency and cost (Harwood, 2015). Despite this, there appear to be barriers to the implementation of healthcare training protocols at virtually every level. The explanation most often given for this is work and safety culture. For this reason, the literature regarding work and safety culture will be reviewed. For safety programs to reach their full potential, a comprehensive commitment from staff at all levels is necessary. These training programs have been shown to reduce the number of injuries effectively and workers' compensation claims, resulting in lower costs for the institution and better outcomes for patients (Howard, 2016; Vollman \& Bassett, 2014). However, two longitudinal studies found that these were not sustained over time (Martin et al., 2009; Theis \& Finkelstein, 2014).

The research gap that will be addressed is to assess the relationship, if any, of repeated Healthcare training sessions, which provide refreshers on safety protocols, thus reducing the severity of healthcare training among direct patient care staff. The researcher wants to determine if the level of work-related musculoskeletal injuries among direct patient care staff are associated with the frequency and/or amount of annual ongoing Safe Patient Handling and Mobility (healthcare training) refresher training among direct patient care staff in the U.S. This information will advance the knowledge in the field by giving policymakers a guide by which they can compare and modify their existing healthcare training training. In the next section, the gap in research is identified, followed by the theoretical foundation for the research. After this, the related literature is discussed considering the recent studies on caregiver injuries and the importance of standardization of healthcare training trainings. The article is concluded with a summary of the discussion.

Identification of the Gap. Little is known regarding the association between ongoing healthcare training refresher training and the severity of healthcare training among direct patient care staff. This calls for the evaluation of leadership ethics within the healthcare industry and the various strategies employed in finding out how to fund and implement ongoing healthcare training refresher trainings for staff members. There is a scarcity of literature focusing on the characteristics of the implementation of healthcare training protocols that impact the effectiveness of the programs. Despite widespread attempts to combat this problem with Healthcare training, healthcare staff are still at high risk for injury, which may be due to a lack of standardization and repetition in Healthcare training programs (Nelson, 2015). Despite policy changes on the safety protocols for hospital staff, little is known whether these policy changes have produced results regarding improving the rate and quality of safety training for direct caregivers (Lee et al., 2015). There is a wide variety of approaches that care facilities have taken to deal with the high injury rate among its staff. Although this is normal in this line of work, these unique occupational hazards can have permanent and career-ending consequences, and exposures to cumulative risk can be detrimental for the staff (Blair \& Bratton, 2015; Clarke, 2017). 
Some explanations for discrepancies proposed by the researchers are inconsistent enforcement of healthcare training protocols by leadership ethics and a lack of "safety culture" in the workplace, which results in poor compliance by staff. The goal of any healthcare training program is to reduce the rate of injury and in direct patient care staff, so it is crucial that the staff employ the techniques that are provided to them during training. However, there is little research on the utilization of the Kirkpatrick Model and how it can provide researchers with contextual analysis. The lack of standardization and repetition of healthcare training poses threats to the individual situations of staff - that is, problems that arise with the implementation of such programs can harm the workers and expose them to a greater risk of injury at the workplace (Charney \& Hudson, 2004; Zadvinskis et al., 2013). As such, the current strategies in healthcare training trainings can be considered as short-term only, and that the effects of such programs are short-lived (Martin et al., 2009; Theis \& Finkelstein, 2014). This connotes that the current methods being utilized for healthcare training trainings are not enough to ensure the overall safety of staff in the performance of their duties. Thus, it is critical to investigate the implementation of such programs and the assessment of their short-term and long-term impact on both the overall healthcare policy and the individual situation and careers of the workers. Although it is known that a cost-effective healthcare training implementation is important, little research is done as to how this can be implemented and how changes in policy can affect the experience of hospital staff (Charney \& Hudson, 2004; Nelson, 2015; Zadvinskis et al., 2013). Standardization of the Healthcare training, while also considering the work and culture of a hospital, may be able to contribute to the improvement of such practices. Furthermore, it was shown that by utilizing an effective healthcare training program, healthcare training were reduced among nursing staff that are responsible for direct patient care (Arnold et al., 2014; Harwood, 2015; Olinski \& Norton, 2017; Powell-Cope et al., 2014; Walker et al., 2017).

\section{Theoretical Foundations and Conceptual Framework}

The theoretical foundation will comprise of one model and one instrument. The model is the Kirkpatrick Level of Evaluation Training, which is used to structure and evaluate training. The instrument is the Oswestry Low Back Pain Disability Questionnaire. Each of these tools supports a variable within this study and is the basis for data collection and development of the research questions.

Kirkpatrick Models of Evaluation. The theoretical framework for this study will be based on the four levels of the Kirkpatrick Model, which includes reaction, learning, behavior, and results. Kirkpatrick (1998) has provided a definition for each of these levels. According to Kirkpatrick, reaction is considered as the degree to which participants react favorably to the training. The reaction area is where the learners give feedback regarding their training. Evaluating reaction is similar to quantifying customer satisfaction. It is imperative to find out how and what people feel about the instruction they received. According to Kirkpatrick (1998), reaction determines how well the person was able to accept the concepts shared within the training.

The second area in the evaluation model is learning. Kirkpatrick (1998) described the importance of this area from some different perspectives. First, people will retain more if they are satisfied with the way they are learning and how conducive the environment is. Learning is considered as the degree to which participants acquire the intended knowledge ("I know it."), skills ("I can do it right now."), and attitudes ("I believe this will be worthwhile to do on the job.") based on their participation in the training event. Second, this evaluation is confirming that learning has taken place because of the training. Without this step being completed before moving on to level three, there is an opportunity for wrong conclusions later as to why the training did not take effect. This step also can identify if the training needs to be improved. Behavior is considered as the degree to which participants apply what they learned during training when they are back on the job. Results are considered as the degree to which the targeted outcomes occur because of the training event and subsequent reinforcement. This is the uppermost level of evaluation within the Kirkpatrick Model (Kirkpatrick, 1998). This determines whether the training had been effective in imparting both knowledge and skills through application. When people can apply ideas or concepts that they learned, it becomes a behavior that they practice on a normal routine. Kirkpatrick also created a 10-step process for developing a training system. Kirkpatrick (1998) Model provided the following ten steps (Ameh \& van den Broek, 2015):

1. Determining ideas;

2. Setting objectives;

3. Determining subject content;

4. Selecting participants;

5. Determining the best schedule;

6. Selecting appropriate facilities;

7. Selecting appropriate instructors; 
8. Selecting and preparing audiovisuals;

9. Coordinating the program;

10. Evaluating the program.

To give the reader an idea of how this model is applied, let us examine its use in another study in more detail. Abdulghani et al. (2014) used the Kirkpatrick Model to evaluate research methodology workshops at King Saud University (KSU). The faculty development unit in the College of Medicine at KSU conducts 35-45 annual workshops for faculty development. This study evaluated five research methodology workshops that took place between 2010 and 2013 using the Kirkpatrick Model. The authors used two evaluations, formative and summative. In the first evaluation, they measured how well the program was implemented; publicizing, organization, delivery of staffing, workshop content quality, educational resources availability, the balance between building knowledge and applicability, the interaction between participants and staff, and feedback on participant performance. The summative evaluation measured the impact of the program on participants, including the acquisition of knowledge and skills, change in the research practices, and changes in their workplace that produced publications. The researchers used both qualitative and quantitative methods for evaluation. Each participant was given several evaluations to complete throughout the workshop. They were given pre-tests to determine their level of knowledge before the workshop so that changes could be measured by a post-test (which addresses the second level of the Kirkpatrick Model, learning).

The authors surveyed participants on the last day of the workshop about their subjective experiences to address the first level of the Kirkpatrick Model, reaction. The researchers asked how they felt about the workshop. The responses were mostly positive, with a few offering suggestions for improvement. To address the second level, learning, the researchers used the results from the pre-and post-test to determine how much knowledge was gained from the program. The authors broke the workshop down into parts and tested each participant on those specific skills. In all aspects, post-test scores were much higher, although for two categories the change did not meet statistical significance. The authors point out that pre-test takers had a good deal of trouble with SPSS, and that was a characteristic that showed the most improvement. In other words, this method measures change, so if the baseline knowledge is already there, the workshop would not add enough knowledge to produce a statistically significant post-test change. A follow-up to the workshop showed that the workshop had indeed produced some behavioral changes (level 3 of the model) and produced some published articles (level 4 of the model). Sixty-six of 116 participants had started a research project, and eight had already been published. While this study does not speak directly to workplace safety, it gives the reader a good idea of how the researcher plans to incorporate the Kirkpatrick Model to evaluate the effectiveness of a training program for direct patient care staff. This model will help to guide the researcher to determine the effectiveness of a training program above and beyond, simply looking at whether participants learned anything. It is particularly useful for evaluating Healthcare training programs because there is some evidence that simply offering a training program or education program is not enough.

It is necessary to evaluate the degree to which direct patient care staff respond well to the training and implement the skills taught. Without this component, the goal of reducing the rate of healthcare training may not be met, even though education programs have been provided. This model also lends itself to the nonexperimental correlational method for this study. Since the researcher is trying to establish whether or not a relationship between the frequency and/or amount of Healthcare training and the severity of healthcare training exists, the frequency or amount of Healthcare training should improve levels 2 and 3 of the model (learning and behavior), which should impact the 4th level, results. In other words, the frequency or amount of SPHM training should improve the knowledge that direct patient care staff have by reinforcing it at multiple intervals. This should impact behavior as more recent Healthcare training is more likely to stimulate the caregiver's mind. This should reduce the severity of healthcare training over time. The first, second, and third levels are crucial to this kind of training because it is imperative that direct healthcare workers not only take in the information but that it permanently alters their behavior. As will be discussed later, there is a good deal of inertia in healthcare. Many nurses and nurse's aides are aging and have a harder time changing the way they do things, so it is important that training programs take into account their reactions to the training (level 1) (American Nurses Association, 2013; Gallagher, 2012; Goetz S.B. \& Taylor-Trujillo A., 2012). While this is not an element that will be explored by this research, it is an important part of evaluating any training program. Second, and perhaps most obvious, the training must increase knowledge. A simple test before training (pretest), immediately after training (post-test), and perhaps at regular intervals afterward can demonstrate how much knowledge is gained by the program. The third level, behavior, is a little more complex. How do you measure what behavioral changes take place after the participants go back to their regular job? This is the hardest part to evaluate, but in this case, it is also the most important. 
The goal of any healthcare training program is to reduce the rate of injury in direct patient care staff, so it is crucial that the staff employ the techniques that are provided to them during training. This can be accomplished a couple of ways. One might survey peers and coworkers to ask them to what extent others employ the techniques used. Alternatively, another option is to combine the last two levels (behavior and evaluation) and use the actual reduction in injury as a proxy to measure the degree to which behavior has been affected. Overall, the Kirkpatrick Model gives the researcher a formula from which to evaluate training at multiple levels, which is particularly useful in this situation. This research will employ the Oswestry Low Back Pain Questionnaire to assess the presence and severity of healthcare training to quantitatively measure the disability level of direct patient care staff (Baker, Pynsent, Fairbank, Davidson, \& Keating, 2002; Fairbank et al., 1980; Fairbank \& Pynsent, 2000; Shameela et al., 2015; Zadvinskis et al., 2013). Due to the nature of the data being gathered, a quantitative method will be employed in this study to measure the relationships between ordinal variables. Per Mujis (2011), quantitative research considers the use of numerical data that are analyzed with mathematically based methods to explain a phenomenon. As opposed to a qualitative study, a quantitative study is focused on answering the degree of relationship between variables (Babbie, 2012).

A quantitative method is used in studies where the objective is to examine relationships between identified study variables (Yoshikawa, Weisner, Kalil, \& Way, 2013) that are quantified or measured with numerical values (Ostlund, Kidd, Wengstrom, \& Rowa-Dewar, 2011). Quantitative studies are deductive and confirmatory, originating from a theory or set of hypotheses that may be validated through statistical testing (Pulido-Martos, Augusto-Landa, \& Lopez-Zafra, 2012). Based on the results of the statistical tests conducted, the researcher can make assumptions about the relationships between the study variables (Yoshikawa et al., 2013). Because the purpose of the study is to explore potential relationships between variables, a quantitative approach is deemed to be most appropriate. Statistical analyses will be used to justify and provide evidence for the existence or the absence of relationships between the identified variables. The extent of the back injury among direct patient care staff is an alarming problem in the healthcare industry (Amin et al., 2018; Chagas, 2016; Forst, Friedman, Chin, \& Madigan, 2015; Sang \& Brings, 2015). The sheer costs to the healthcare industry regarding LWD's, WC claims, and attrition are staggering (Chiwaridzo, Makotore, Dambi, Munambah, \& Mhlanga, 2018; Sang \& Brings, 2015). Despite the well-documented costs of healthcare training to healthcare, this problem continues to persist due to the lack of policy changes that focus on workplace safety (Harwood, 2015; Sang \& Brings, 2015). For instance, the higher bed capacities and increased incidence of obesity in patients have increased both the frequency and intensity of physical demands on caregiving staff (Sang \& Brings, 2015). These have increased both the frequency and intensity of lifting, bending, and twisting, which puts direct patient care staff at even higher risk for healthcare training. This reflects the need to establish protocols on how to properly handle increased workloads through direct care staff and utilizing the proper healthcare training equipment.

It has been documented that the frequency and intensity of lifting demands on direct patient care staff regularly exceed the 35lb limit recommended by the National Institute for Occupational Safety and Health (NIOSH) (Arnold, Combs, Gach, \& Labreche, 2015). One meta-study of other research found that lifting more than $25 \mathrm{~kg}$ (55lbs) at one time or lifting at a frequency of $25+$ lifts a day increases the incidence of LBP by $4.32 \%$ and $3.5 \%$, respectively. These findings coincide well with other research and with healthcare training guidelines for lifting during work (Coenan et al., 2016). These statistics, combined with a lack of lifting equipment, give us some insight into why direct care staff frequently engage in risky work practices that lead to high rates of injury. Another factor that may contribute to the ongoing epidemic is underreporting of back injuries by direct patient care staff. The reasons for underreporting and going to work despite the presence of healthcare training are multifaceted, but the common characteristic here is workplace culture. For instance, many healthcare staff fear reprisals or being labeled a complainer. Some nurses work through temp agencies, so they feel too powerless to say anything. Some direct patient care staff blame themselves for their injury and so forgo reporting the injury or continue working despite their pain. This research agrees with other research which found a connection between work stress and the presence of healthcare training (Ykoyama, Hirao, Yoda, Yoshioka \& Sherakami, 2014).

Other research has suggested that healthcare staff burnout can contribute to underreporting. All the above evidence suggests that workplace culture is an issue worth exploring, and that is a topic that will be discussed in more detail later. The costs of injury in the healthcare profession are huge, both in terms of nominal costs and human costs (Williams, Penkala, Smith, Haines, \& Bowles, 2017). Even though this information has been around for quite some time, the situation appears to be getting worse rather than better. The increase in concern could either be the result of increased awareness or the increasing age of the care staff. Either way, at the very least, healthcare training among direct patient care staff does not appear to be a problem that is being 
adequately addressed. Statistics from the healthcare training reported above show that concerns appear to match reality in terms of the incidence of injury. The situation does not appear to be improving. The current research aims to help the healthcare community in understanding what measures can be taken to improve compliance with healthcare training protocols.

State Policies and Grassroots Campaigns. Several states have passed laws regarding healthcare training standards (Menzel, 2008; Monaghan, 2013; Powell-Cope \& Rugs, 2015; Rockefeller \& Weaver, 2016). The laws vary in the type and extent (Perez, 2016), and are recent additions to the occupational landscape. There is little research to assess the effectiveness of these laws, but a couple of studies have been completed with some conflicting results. The laws that have been passed are very recent (with the oldest legislation going back to only 2005), so long-term assessment of their impact is somewhat limited. One study (Rockefeller \& Weaver, 2016) that surveyed 379PTs in three states with legislation detailing healthcare training standards, Washington, California, and Oregon, found that only $45 \%$ reported having a healthcare training program in place. However, the survey return rate was quite low, which indicates that many of those surveyed with Healthcare training programs may not have responded. Two additional studies looked at specific states, one in Washington and one in California.

Washington passed legislation in 2006, ESB1672, which covered acute care facilities in the state. While the law was implemented in 2006, it was phased in over several years, giving facilities until 2010 to meet all the requirements. One unique feature of this legislation is the requirement that the Department of Labor and Industries (DLI) conduct a post-implementation study to determine the effect of the legislation on healthcare training claims for hospitals with Healthcare training programs. Shortly after implementation, the DLI found a decrease in healthcare training claims in hospitals, and four years after full implementation, those claims had continued to decline (Howard, 2016). In 2012, California passed Safe Patient Handling legislation that requires hospitals to implement safe patient handling protocols in 2012, while healthcare training adopted regulatory standards in 2014. The law requires the replacement of manual lifting policies with lift teams and lift equipment in acute care facilities in California. It is important to note that the law applies only to acute care facilities. This is an important point, as many direct patients' caregivers work in other occupational settings, such as nursing homes and rehabilitation facilities that carry a similar level of risk. Lee et al. (2015) intended to assess the effectiveness of this legislation in reducing the presence of WRMIs in healthcare staff.

Despite the implementation of healthcare training legislation in California, the 12-month prevalence rate of healthcare training was still about $70 \%$. Only $33 \%$ of respondents reported changes to their hospitals' policies after the legislation. The authors (Lee et al., 2015) concluded that while the legislation had improved practices somewhat, more steps should be taken to ensure that hospitals fully comply with safety standards $100 \%$ of the time. It should also be noted that while many nurses work in acute care facilities, the risk of healthcare training is also very high in many other kinds of caregiving environments, particularly in long term care facilities. So, while healthcare training legislation is a step in the right direction, it does not address the much larger issue of injuries and associated costs for the entire industry.

The ANA standards set forth break up responsibilities for healthcare training into two broad categories; the responsibilities of the employer and those of the staff. There are eight overarching standards of care:

1. The Culture of Safety: a collective and sustained commitment by everyone within the organization to emphasize safety over competing goals.

2. Sustainable healthcare training Program: a formal, systematized healthcare training program for reducing the risk of injury for healthcare recipients and healthcare workers.

3. Ergonomic Design Principles: a systemized proactive approach that includes prevention considerations in all designs that affect individuals in the occupational environment.

4. Healthcare Training Technology: Assistive tools available at the point of care to facilitate HEALTHCARE TRAINING. Technology can include equipment, devices, accessories, software, and multimedia resources.

5. Education, Training, and Maintaining Competence: a system of training and education to maintain the healthcare training competence of healthcare workers who provide direct care.

6. Patient-Centered Assessment: the plan of care adapted to meet the healthcare training needs of individual healthcare recipients and specify appropriate healthcare training technology and methods.

7. Reasonable Accommodation and Post-Injury Return to Work: a comprehensive healthcare training program that can help employers provide reasonable accommodations to healthcare workers who were injured. 
8. Comprehensive Evaluation System: a system to evaluate healthcare training program status, using staff performance, staff injury incidence, and severity, and healthcare recipient metrics (American Nurses Association, 2013).

Powell-Cope and Rugs (2015) reviewed the legislation passed in several states and highlighted the degree to which states had adopted the recommendations set forth by the healthcare training in their guidelines. The states reviewed were Texas, Washington, Hawaii, Ohio, Rhode Island, Maryland, New Jersey, California, Illinois, Minnesota, and New York (Powell-Cope \& Rugs, 2015). The only guideline to be universally adopted was one requiring education and training regarding healthcare training. Ten states require formal Healthcare training programs, while all but eight states in the study have implemented evaluation standards, six states have adopted the ergonomic design, technology, and patient care requirements, and only five states have adopted standards for post-injury return to work. Each of the eight standards set forth by the healthcare training also stipulated specific recommendations for each, the discussion of them specifically is beyond the scope of this paper. However, the degree to which the specific standards were adopted varies by state (Powell-Cope \& Rugs, 2015).

Policy changes are critical in promoting safety in the workplace (Purnomo, Giyono, \& Apsari, 2017; Taderera, Heinrich Hendricks, Pillay, \& Hendricks, 2017). Taderera et al. (2017) argued that it is necessary to examine the effect of healthcare reform policies in fostering a universal health coverage goal for healthcare staff. A total of 87 healthcare workers participated in semi-structured interviews. The data from the interviews were analyzed together with the qualitative data from documentary research, five key informant interviews, seven in-depth interviews, and five focus group discussions (Taderera et al., 2017).

Three factors were identified, which are: (a) deployment of community health workers, (b) financial incentives, and (c) safety, health welfare, and salaries. In the absence of legislation, some nurses have taken it upon themselves to initiate change. In Northwestern Lake Forest Hospital (NLFH), nurses were increasingly concerned about injury due to increased workloads and more complex patient care needs. In this observational study, the researchers looked at the steps taken by the nurses and the hospital staff to implement a healthcare training program from the bottom up. They describe what they believed to be the crucial components of a grassroots campaign to improved training within the institution. The nurses first wanted to gather research related to Healthcare training programs and their relative effectiveness, so they collected data to make their case to the leadership ethics of the hospital. Once that was done, they reached out to a nurse researcher to help guide the process of implementing a study in their hospital. Support from the leadership ethics of the hospital is essential for promoting a healthcare training program from within. The researchers demonstrate that a healthcare training program can be implemented from the bottom up and that it is important to engage leadership ethics in the implementation of any major initiative in a large organization. This study sought only to describe the implementation of the program and does not discuss any results as far as reducing the number of healthcare training on the staff, but it is an interesting case that suggests a strong safety culture is something that can be implemented from the bottom.

The levels of initiation - both government and grassroots - can and have produced changes in policy in critical care units across the country. However, it is obvious from this discussion that healthcare training policies are hardly the standard, and only six states have applied their legislation to all healthcare facilities. While California, Washington, and Illinois have taken some steps, the legislation only applies to hospitals, which misses a large number of direct patient care workers in other facilities (long term care, home healthcare, occupational, and physical therapy facilities). However, just because some states have not passed legislation requirements, does not mean care facilities are doing nothing. Many institutions across the country have implemented Healthcare training programs and interventions to reduce injuries and decrease healthcare training costs. However, there is no industry standard about what constitutes healthcare training program. The next section explores those safety interventions in detail and the extent to which they reduce injuries and their associated costs.

Work-Related Injuries and Healthcare Workers. Work-related musculoskeletal disorders and injuries are prevalent in the healthcare industry (Gomaa et al., 2015; Salamanzadeh, Rahimi, Goshtasbipour \& Meripoor, 2016; van der Beek et al., 2017). Workplace conditions, activities, and equipment put the healthcare workers at-risk of temporary and long-term injuries (Salamanzadeh et al., 2016). Despite this, Stock et al. (2018) argued that there is little evidence on the effectiveness of interventions in preventing work-related injuries and disorders. The frequency of work-related musculoskeletal injuries and disorders is considered an "impending epidemic" in the healthcare industry (Epstein et al., 2018). Understanding the various factors and variables associated with healthcare training entails analyzing the impact on the physical and mental well-being of healthcare workers (Amin et al., 2018; Chiwaridzo et al., 2018; Dabholkar, Yardi, Dabholkar, Velankar, \& 
Ghuge, 2017; Stock et al., 2018; Vieira et al., 2016). For instance, Vieira et al. (2016) found that up to 90\% of PTs have work-related musculoskeletal disorders (WMSD) during their careers, while $50 \%$ have experienced healthcare training within the first five years of their practice. The lower back was the main body part that was affected (Vieira et al., 2016). PTs who are female and who those who work in hospitals are more susceptible to healthcare training (Vieira et al., 2016). It was also found that healthcare training are associated with age, gender, specialty, and job tasks (Vieira et al., 2016). Manual therapy, lifting, and transferring of patients were found to cause the development of healthcare training among PTs (Darragh \& Campo, 2017; Rockefeller \& Weaver, 2016; Vieira, 2016).

Work-related injuries among healthcare workers are rampant and can influence the over-all work experiences of the individual (Chiwaridzo, Makotore, Dambi, Munambah, \& Mhlanga, 2018; Epstein et al., 2018; Kanaskie \& Snyder, 2018; Salmanzadeh et al., 2016). In a meta-analysis focused on surgeons and interventionalists, Epstein et al. (2018) investigated the prevalence of healthcare training to assess the preventive efforts required to minimize such incidents. Data was gathered on disease prevalence of the neck, shoulder, back, and upper extremity, and measures to address such problems were gathered (Epstein et al., 2018). Among the 21 articles reviewed, with a total sample of 5,828 physicians and 14.4 hours of procedures per week, prevalence estimates showed that $17 \%$ suffered degenerative cervical spine disease, $18 \%$ had rotator cuff pathology, $19 \%$ had degenerative lumbar spine disease, and 9\% suffered from carpal tunnel syndrome (Epstein et al., 2018). Epstein et al. (2018) also found that the interventions for these disorders and injuries were focused on products and behaviors and that the main reason for the prevalence of healthcare training was the lack of awareness and training on ergonomics. These numbers reflected the alarming threat of work-related injuries on the careers and well-being of healthcare workers. It is further recommended that an evidence-based ergonomics program be investigated to evaluate the efficiency of such programs in minimizing and preventing career and lifethreating injuries in the workplace (Epstein et al., 2018).

In another study, Salmanzadeh et al. (2016) examined the prevalence of needle-stick injuries and factors associated with them. Healthcare workers in Iran participated in this cross-sectional study (Salmanzadeh et al., 2016). The study was divided into two parts: the first part was focused on analyzing the demographics of the participants, while the second part highlighted the records and number of needle-stick injuries in the span of one year (Salmanzadeh et al., 2016). It was found that nurses experienced the highest frequency of needle-stick injuries, and such injuries happen more frequently during night shifts. Salmanzadeh et al. (2016) posited that vaccination, comprehensive screening, and appropriate training are critical in minimizing and preventing such accidents. Likewise, Singh and Paudel (2015) argued that education and interventional strategies should be the priority in addressing needle-stick injuries. It was also found that the highest prevalence of needle-stick injuries happens among nurses (Singh \& Paudel, 2015). As such, the pervasiveness of work-related injuries is a matter that must be addressed through proper education, training, and informed interventions (Isara, Oguzie, \& Okpogoro, 2015; Salmanzadeh et al., 2016; Singh \& Paudel, 2015).

Healthcare training and injuries are also associated with the emotional well-being of healthcare workers (Amin, Quek, Oxley, Noah, \& Nordin, 2018). Amin et al. (2018) investigated emotional distress as a predictor of healthcare training among nurses. A total of 660 female nurses in public hospitals participated in the selfadministered survey (Amin et al., 2018). It was found that anxiety and distress were significantly and positively associated with the risk of work-related disorders and injuries (Amin et al., 2018). Amin et al. (2018) suggested that it is important to understand the occupational and non-occupational sources of distress and anxiety among nurses. This reflects the study by Chagas (2016), which showed that the gradual development of work-related disorders and injuries is related to the repetition of tasks, emotional distress, excessive strain due to inappropriate posture, and psychosocial risks. A survey was conducted on 86 home care service workers regarding the prevalence and symptomatology of musculoskeletal problems (Chagas, 2016). It was revealed that symptoms of musculoskeletal disorders are high in anatomical areas such as the upper limbs and the lumbar, dorsal, and cervical areas (Chagas, 2016). These studies demonstrate the threat of work-related disorders and injuries and its association with the emotional and psychological well-being of the healthcare workers (Amin et al., 2018; Chagas, 2016). As such, it is critical to include interventions that address the psychological health of the workers (Chagas, 2016).

Safety Interventions on Work-Related Musculoskeletal Problems. The type and scale of safety interventions vary a great deal by the institution (Purnomo, Giyono, \& Apsari, 2017; Taderera, Heinrich Hendricks, Pillay, \& Hendricks, 2017; Williams, Haines, Penkala, Smith, \& Bowles, 2017). Hospitals have increased the availability of lifts, instituted training sessions, organized lift assistance teams (LAT), and implemented exercise programs to try to combat the increasing rate of a low back injury in its staff. Despite 
the wealth of information available regarding the prevention of injury, many hospitals and staff still employ techniques based on personal experience. One type of intervention employed is the lift assistance team (Schoenfishch et al., 2011). These are teams, drawn from many parts of the hospital, that are trained as a group to move patients. This shifts the burden of moving patients from a single caregiver to a group that specializes in moving and lifting patients. In one hospital where LATs have been employed, nurses report increased staff safety and work satisfaction. It is, however, not clear if using LATs reduces the rate of injury, or simply shifts it to another group in the hospital (Schoenfishch, Lipscomb, Myers, Fricklas, \& James, 2011). Further research is needed to determine the effectiveness of this intervention for reducing the number of injuries.

Ergonomics is one of the most studied and pervasive interventions for work-related musculoskeletal problems (Purnomo, Giyono, \& Apsari, 2017; Taderera, Heinrich Hendricks, Pillay, \& Hendricks, 2017), which could help healthcare workers in manual labors required on the job (Oranye \& Bennett, 2018). Ergonomics programs aim to limit intensity, duration, and frequency of lifting during caregiver shifts to reduce the incidence of back injury and LBP (Purnomo et al., 2017; Taderera et al., 2017; Wanless \& Wanless, 2015). For instance, Wanless \& Wanless (2015) published a paper with recommendations regarding the proper use and implementation of lifting procedures to reduce injury. Wanless and Wanless (2015) argued that hoisting equipment should reduce injury, but that despite its use, injuries continue still occur. This is likely due to poor techniques and improper training (Wanless \& Wanless, 2015). This provided evidence that repeated training could reduce the number of injuries significantly, even in facilities that have taken some steps to improve safety. Furthermore, documentation and feedback are critical and that even close calls should be reported to improve safety protocols (Wanless \& Wanless, 2015). The use of ergonomics in addressing musculoskeletal injuries and disorders has been studied in various contexts (Al-Sabah, Al Haddad, \& Khwaja, 2018; Cheng, Ju, Yu, \& Wong, 2016; Oranye \& Bennett, 2018; Purnomo et al., 2017; Williams, Haines, Penkala, Smith, \& Bowles, 2017). For instance, Purnomo et al. (2017) suggest that a macro-ergonomic work design may be utilized in reducing musculoskeletal disorders and injury risk in training. Improvement of job competence entails integrating ergonomic design, which included adjustable seats, embroidery hoops tables with a footrest, improved lighting, facemasks, earplugs, and a drinking water facility (Purnomo et al., 2017). The application of an ergonomic design demonstrated a $60.39 \%$ reduction of musculoskeletal problems and a $22.2 \%$ reduction in the risk of injury (Purnomo et al., 2017). Such results reflect the effectiveness of ergonomics and may also be applied in the healthcare profession.

In another study by Hsin-Yi Kathy Cheng, Man-Ting Wong, Yu-Chung Yu, \& Yan-Ying Ju (2016), the workrelated ergonomic-associated variables and its relation to work-related musculoskeletal problems were investigated among healthcare professionals focused on special education. A total of 338 special education schoolteachers and teacher's aides answered a questionnaire focused on three domains, which included demographics, the prevalence of work-related musculoskeletal problems, and ergonomic factors (Cheng et al., 2016). Among the sample, 89\% reported having had experienced musculoskeletal disorders, with the most affected anatomical parts being the lower back, shoulder, and wrist. Results of the regression analysis showed that background factors, students with multiple disorders, and ergonomic factors such as assistance in diaper changing and other duties were strongly linked to the development of work-related healthcare training (Cheng et al., 2016). Also, the use of assistive devices was found to reduce the prevalence of work-related MSDs (Cheng et al., 2016). Likewise, Williams et al. (2017) argued that there is a need for policies within healthcare facilities to consider how to mitigate healthcare professionals' musculoskeletal health. The role of staff in providing healthcare makes them susceptible to musculoskeletal health (Williams et al., 2017). Williams et al. (2017) investigated the risk factors in the podiatry profession. Results showed that out of the 948 respondents, $29 \%$ reported that the body area targeted was the lower back. Being female was also significantly associated with higher back pains. Four themes related to musculoskeletal pain emerged: (a) organizational and procedural responses to injury, (b) giving up work, taking time off, reducing hours, (c) maintaining good musculoskeletal health, and (d) environmental change. These studies demonstrate the effectiveness of evidence-based ergonomics methods integrated into the healthcare workplace to address the prevalence of musculoskeletal injuries and disorders among workers (Cheng et al., 2016; Williams et al., 2017).

Oranye and Bennett (2018) examined the musculoskeletal and non-musculoskeletal injuries among workers in the healthcare sector to understand the implications for work disability management. Using secondary data from cohort workers between 2002 and 2012 healthcare training Manitoba, it was found that idiopathic workrelated musculoskeletal disorders (WMSD) comprised of $74.6 \%$ of the injury claims, while a significant difference among females were found in terms of the injury risks (Oranye \& Bennett, 2018). There were significant differences among occupational groups in terms of the prevalence of musculoskeletal injuries (Oranye \& Bennett, 2018). The frequency of physical activities required on the job increases the risk of 
musculoskeletal injuries among direct patient care staff (Oranye \& Bennett, 2018; Williams et al., 2017). Several studies have shown the use of lifting equipment can reduce the rate of injury and healthcare training in healthcare staff (Elnitsky, Lind, Rugs, \& Powell-Cope, 2014; Mayeda-Letourneau, 2014; Mechan, 2014; Wanless \& Wanless, 2015; Olinsky \& Norton, 2017). One long-term study of patient handling devices and a comprehensive ergonomics program took place in six long-term care facilities (LTC) and one chronic care facility $(\mathrm{CCH})$. They compared pre-and post-implementation rates of injury and lost workdays for several years after implementation and found a sizable impact, with a 59.8\% reduction in injuries and reduction in workers' compensation costs by $90.6 \%$. This study used a similar approach to the current proposed study, in that the researcher will attempt to identify if there is a relationship between the frequency and amount of ongoing healthcare training refresher training and the severity of healthcare training.

Alamgir et al. (2015) conducted a longitudinal study to determine the impact the use of lifts had on the incidence of healthcare training. They examined the six-year period before introducing the lifts with the four years after introduction in three long term care facilities. They found a substantial and sustained decrease in workers' compensation claims and days lost per bed. Alamgir et al. (2015) also noted that the hospital could recoup its investment in six years if only direct costs were considered, and two years when indirect costs were added. This provided support to the importance of implementing lifting equipment in hospitals, although there does appear to be some resistance to the upfront cost. This study demonstrates that those costs can be recouped quickly. However, given that some studies have shown injuries to persist despite the presence of lifting equipment, proper training is also necessary for the lifts to be effective (Wanless \& Wanless, 2015). Hospital investment on equipment does not necessarily mean that these machines are utilized, as reflected in the data from the 2013 ANA survey on health and safety. Respondents reported that even though lift equipment was more widely available in 2011 than in 2001 (indicating that workplaces had invested in its procurement), nurses reported using it frequently when available less than half the time (American Nurses Association, 2013). One issue raised in the literature regarding the use of lifting equipment is the effect this may have on patients, both regarding treatment outcomes and psychological stress of being lifted with equipment. Some patients may find the use of some of this equipment embarrassing, which can affect their psychological health. Furthermore, some authors have noted that care must be taken to ensure that the use of equipment does not cause harm to the patient (Peterson, Kahn, Kerrigan, Gutmann, \& Harrow 2015; Elnitsky et al., 2014). Since the equipment is large and complex, it is imperative that caution is taken to make sure that it does not cause harm to the patient or the caregiver. It is also imperative that operators have complete safety training regarding the use of the equipment to ensure that it is used properly.

However, it seems that concerns regarding patient outcomes have been largely unfounded. For some patients, mobility is key to their recovery, and when used properly, lifting equipment can improve mobility (Arnold, Wilson, McIlvaince, Labreche, \& Stevens, 2015). This issue has become a larger concern as the rate of obesity has increased dramatically in recent years, making bariatric care another part of the equation. This is complicated by the fact that improving mobility as a part of the treatment plan is both necessary and dangerous for healthcare staff. However, using lifts to move larger than average patients can conflict with the compassion centered culture of caregiving, which can lead nurses to attempt to move these patients manually. While this may appear at first to improve the situation, it increases the danger to both the patient and the caregiver. This is further complicated by the fact that patient lifting equipment is not always available, and when it is, it is not always employed (American Nurses Association, 2013; Gallagher, 2015). While mobility is an issue for geriatric centers, technology can help to overcome these barriers to bariatric care (Arnold, Combs, Gach \& Labreche, 2015). This has a twofold benefit; it improves the safety of the staff and increases the mobility of the patient, which may be a necessary part of the patient's recovery. Lifting equipment, healthcare training, and ergonomics program all have positive impacts on injuries and WC claims. While these methods are widely employed by themselves, they are more often the part of a larger initiative for healthcare training that involves some training and knowledge assessment. In the next section, we will discuss attempts to increase the awareness of nurses and nursing staff about safe patient protocols and proper lifting techniques.

Safe Patient Handling and Mobility Programs. Far more common than single intervention approaches are Healthcare training programs that employ a variety of techniques to address issues of healthcare training, injury, worker absenteeism, and healthcare training. Research has shown that multi-pronged approaches are far more effective than single intervention approaches (Bonomi et al., 2017; Enos, 2013; Latvala \& Masterman, 2017; Olinski \& Norton, 2017; Rockefeller \& Weaver, 2016; Walker et al., 2017). Case studies of implementation have shown that they can be very effective at reducing injuries and their associated costs (Bonomi et al., 2017; Kennedy \& Kopp, 2015; Latvala \& Masterman, 2017; McKinney, 2015; Walker et al., 2017). While it may seem obvious because of the risk of injury, education and training on healthcare training protocols 
are not as widespread as one might hope. There are large variations in knowledge among staff and training, and education programs are not thorough in this regard. This section will explore these issues in more detail. Kay \& Glass (2011) did a study that suggests that healthcare training protocols may not be as well-known as could be assumed. They surveyed 100 nurses in an Australian private hospital about their subjective level of safety knowledge. They then tested the respondents for actual safety knowledge. They found that while $82 \%$ of respondents claimed to use safe manual handling practices, only $18 \%$ correctly answered questions about safe handling. This suggests there is a gap in knowledge among nurses between what they think they know and what they know. It also suggests that one-way hospitals can improve safety is simply by improving awareness of safety protocols. Indeed, several studies have shown, using pre-and post-test design, that training programs are effective at increasing the knowledge of proper healthcare training protocols (Bonomi et al., 2017; Latvala \& Masterman, 2017; Wilson et al., 2011). These studies address the first level of the Kirkpatrick Model, learning.

There is, however, a qualification to this last claim. That is, while a pre-and post-test design shows how much someone learned from a class, it does not tell if they sustain that knowledge over time or if it modifies their behavior more permanently. This addresses the second level of the Kirkpatrick Model, behavior. A study by Theis and Finkelstein (2014) suggests that this may not be the case. The staff took the rate of injury for a period of 2.5 years after a course on healthcare training. They found an initial dip in the report of injury until, but the rate returned to baseline by the end of the period. This suggests that retraining is necessary to sustain improvements, although how often and to what extent remains to be determined. That is the goal of this research, to determine how often ongoing healthcare training refresher training must be implemented to modify behavior successfully and to maintain that modification permanently.

Oakman, Rothmore, \& Tappin (2016) note that all Healthcare training programs are not equally successful. They argue that part of the reason some efforts fail is that they attempt to use a one size fits all approach and fail to consider certain aspects of their organization. For example, Gallagher (2012) argued the Healthcare training programs should consider intergenerational differences before implementation. The author argued that the cultural differences between the generations mean that program implementation should be handled differently. This is one kind of consideration that may affect how a program is implemented, but others should consider the facility and other employee characteristics to tailor an approach that will stick (Oakman et al., 2016). This recommendation fits well with the first element of the Kirkpatrick Model. It is important to gauge the reaction of the participants so that the information is well received. Otherwise, it will limit the changes in learning, behavior, and results, the last three levels of evaluation in the Kirkpatrick Model. Olson et al. (2016) studied the effectiveness of the Community of Practice and Safety Support (COMPASS) Total Worker Health intervention for home care workers. A total of 16 clusters of workers $(n=149)$ participated in the 12 -month intervention of scripted and peer-led, and involved education on safety, health, and well-being; goal setting and self-monitoring; and structured social support. It was found that relative to control produced significant and sustained improvements in workers' experienced community of practice (Olson et al., 2016). Additionally, significant improvements were found as the use of ergonomic tools or techniques for physical work, safety communication with the consumer-employers, hazard correction in homes, fruit and vegetable consumption, lost workdays because of injury, high-density lipoprotein cholesterol, and grip strength. Olson et al. (2016) concluded that COMPASS was effective for improving home care workers' social resources and simultaneously impacted both safety and health factors.

Singh and Paudel (2015) posited that appropriate education and interventional strategies are critical in ensuring safety in the workplace. We have seen that Healthcare training programs can be quite effective at reducing injuries and their associated costs. However, training programs should consider specific facility and staff characteristics. Programs should address a multitude of factors, including education, ergonomics, lift equipment, and healthcare training. One other issue remains to be addressed, however. If this is such a pervasive problem, one would expect education programs to emphasize safety and proper handling. The literature shows that direct care education programs teach healthcare training protocols at varying levels and with varying levels of emphasis. This may help explain why, despite widespread knowledge, rates of injury and healthcare training continue to be a problem. It also suggests that regular training by the institutions can go a long way in reducing injuries and their associated costs. That is the subject of the next section.

Nursing and Caregiver Education. Nursing education programs have been slow to adopt evidence-based programs to teach students about staff safety. Indeed, nursing students report feeling low back pain because of their on-the-job training at an alarming rate (Knewafsey, Ramsay, Edwards \& Callaghan, 2012). This also appears to be the case with students as well (Stevenson et al., 2011). One study found that exposure to tertiary 
study for two years resulted in an increased incidence of LBP. This is alarming because the onset is so early in the careers of these healthcare workers, which may contribute to attrition and turnover in the field. While manual handling techniques seem to be the standard, evidence-based techniques should take priority. This suggests that either they are not getting the proper information, or the information they are getting is not properly modifying their behavior. Many nursing students report feeling low back pain during their student placements (Menzel, Feng \& Doolen, 2016). One reason for this may be non-ergonomics-based education, relying too much on "good body mechanics" to prevent injury. Several studies have confirmed that students do not get adequate safety training, and those that do receive training may be getting bad information. Most programs reported a mix of manual and safe patient practices. It is unclear from the literature why this is the case. If there is substantial evidence that healthcare training protocols are much more effective than manual handling, why do education programs fail in this regard? The literature does not offer a good explanation for this conundrum and could provide an avenue for another research study.

This makes a strong argument for improving the availability of evidence-based approaches in hospitals and improving nurse awareness through information and training. To be sure, some hospitals have taken on this approach and implemented programs to help increase the knowledge of its staff regarding safety procedures (Bonomi et al., 2017; Olinski \& Norton, 2017; Walker et al., 2017). However, it is unreasonable that so many healthcare staff enter the profession so ill-equipped to deal with the most basic safety protocols to protect themselves from injury. One takeaway from this review is that an educational institution can and should provide a much better curriculum related to healthcare training than is currently offered. In general, it is somewhat of a conundrum that a discipline dominated by science is so scientifically behind in this department, but that is the subject of another study. Lemo et al. (2012) reviewed the literature regarding the implementation of knowledge awareness programs and exercise courses and found that both can be effective in reducing the rate of injury. While these programs have shown effectiveness, we also know that these reductions in injury may not be sustained over time. It is the subject of this study to determine how often these programs must be attended to maintain their benefits. The research also shows, "no lift" policies have been implemented at many hospitals that have invested in patient lift equipment. As discussed previously, these pieces of equipment have a variety of functions; some are sitting-to-stand; others are ceiling lifts (Thomas-Olson, Gee, Harrison, \& Helal; 2015). They are designed to prevent the staff from lifting loads that are too heavy. These programs have shown a good deal of success in reducing staff injury (Nelson et al., 2006; Wanless \& Wanless, 2015).

Van der Beek et al. (2017) further argued that the development and implementation of prevention strategies entail focussing on physical risk factors such as manual lifting and awkward postures. Despite programs to prevent or reduce musculoskeletal disorders (MSD), these have not been successful in reducing the burden because of insufficient knowledge of etiological mechanisms and/or the scarcity of inappropriate and feasible interventions (van der Beek et al., 2017). Also, the prevalence of disorders among staff performing demanding tasks is still not known (Vieira, Schneider, Guidera, Gadotti, \& Brunt, 2016). To summarize, the literature is quite clear. Since Healthcare training programs have been shown to reduce injury greatly, they should be a standard part of any caregiver education program. This does not seem to be the case. One thing that could be done, it would seem, to improve the risks associated with this kind of work would be to improve knowledge and safety education before healthcare staff enters the workforce. Improving the national curriculum for healthcare staff would be a massive undertaking.

Further, as mentioned above, some research has shown that one shot education programs only work for a while before employees return to standard practices. This suggests that while improving education may help, it is not the primary solution to the problem. Furthermore, this would only help future healthcare staff and does nothing for the older generations currently working. This makes another strong argument for implementing widespread, ongoing healthcare training programs within all caregiving institutions. The cost of WMSI's and LBP in terms of both money and human cost is extraordinary. The literature has shown that caregiving facilities have a good deal of tools at their disposal to reduce the rate and severity of the injury as well as the massive amount of costs that go with them. So why have more facilities not adopted these approaches, and for those that have, why are they not always as effective as they could be? That is the topic of the next section.

Work Culture and Behavior. Few studies have focused on the safety culture in the workplace (de Wet, Johnson, Mash, McConnachie \& Bowie, 2012; Edwards, Davey \& Armstrong, 2013; Eriksen, Bruusgaard \& Knardahl, 2004). The definition of safety culture in the workplace is still debated, and very few details are available. Although several studies cite culture as a possible influence on the outcomes of healthcare training protocols, the concept of safety culture seems to use the subjective experience of nursing staff as a measure of safety culture. As such, Edwards, Davey and Armstrong (2013) give us a much more thorough discussion of 
safety culture and its implications. Their focus is on the behavior of those in the workplace: Safety is viewed as the combination of those behaviors which either increase or decrease the risk of harm, with safe denoting protected from harm, and unsafe at high risk of harm. Two different workplaces, even though they perform the same work, can have a remarkably different organizational culture that contributes (or undermines) the work practices of both the leadership ethics and the employees (Kanaskie \& Snyder, 2018). The concept of safety culture is simply a logical extension of that concept whereby members of the organization, through their behavior, thoughts, beliefs and coping strategies create an environment whereby those behaviors, thoughts, and beliefs are passed on to new members of the group and are constantly reinforced by all members of the organization (Edwards, Davey \& Armstrong, 2013). The literature surrounding safety culture and Healthcare training programs is somewhat scattered, but many studies refer to something that resembles work culture or climate (Celona, 2014; Enos, 2013; Harris, 2013; Vollman \& Bassett, 2014). Some studies refer to "social support" to elucidate the importance of leadership ethics preferences in behavioral outcomes. The perception of a safety climate, however, may depend on the relative position one occupies in an organization.

For example, de Wet et al. (2012) conducted a survey of over 400 team members from 49 practices in Scotland and found that perceptions of the safety climate vary within the organization depending on the position of the participant and a couple of other factors like experience, community vs.practice-based, and their professional roles and training status. The most significant variation was between staff groups, indicating that the perception of safety climate may be different depending on who is asked. While it may not appear on the surface that work culture could impact the rate of injury or improve the practice of safety, research suggests quite the opposite. Eriksen, Bruusgaard, \& Knardahl (2004) conducted a quite extensive longitudinal study designed to measure the impact of certain workplace characteristics on the incidence of healthcare training among nurses' aides. The researchers noted that low job satisfaction and low social support at work have both been empirically linked with the incidence of healthcare training. While some studies have explored the relationship between psychological factors and LBP, none have looked at nursing personnel, and the results have been mixed. The researchers wanted to assess which work characteristics were associated with the incidence of healthcare training. They surveyed 4,266 nurses' aides that were either not bothered or only a little bothered by healthcare training during the previous three months and not on sick leave while completing the questionnaire in 1999. Three thousand eight hundred eight of those participants completed a follow-up survey three months later, and 3,651 of those completed another follow-up 15 months later.

Sick leaves and intensity of healthcare training were assessed through the follow-up. The surveyed contained questions about workload and frequency of lifting and twisting and included a measure regarding the work environment called the General Nordic Questionnaire for Psychological and Social Factors at Work "QPSNordic." It assesses work characteristics like social support, perceived fairness, leadership ethics, and organizational culture. The measure of healthcare training was those that reported healthcare training of high intensity or took an LBP-related sick leave that lasted longer than three days at the three-month follow-up. At the 15-month follow-up, the outcome measure was healthcare training related sick leave lasting longer than 14 days and the proportion that reported sick leaves longer than eight weeks. What they found is that healthcare training was predictably related to the degree and frequency of loads lifted. More interestingly, intense healthcare training was associated with a perceived lack of support from immediate supervisors and perceived a lack of pleasant and relaxing culture in the workplace. Long term healthcare training-related leaves were associated with a perceived lack of supportive and encouraging culture at work, working night shifts, and working in a nursing home.

This study is particularly interesting because the authors accounted for previous health conditions, previous low back pain, and several other confounding variables and still find predictive validity in the measures of what can arguably be called workplace culture. It could be the case the incidence of injury is higher at night and in nursing homes because supervision is more limited, and staff are less likely to follow procedures to the letter. In any case, this study provides evidence that workplace characteristics can contribute to LBP and its associated costs (sick leaves, healthcare training, and turnover). The results of this study are further supported by Pekkarinen et al. (2013). The authors wanted to evaluate how workplace characteristics moderate the relationship between heavy workloads and musculoskeletal symptoms. In other words, holding the workload constant, does environment matter? The short answer is yes. The authors found that self-reported physical workload was associated with musculoskeletal symptoms for nurses with low social support. Additionally, the mental workload was associated with a higher risk of musculoskeletal symptoms. Taken together, these two studies suggest that there is a connection between psychological characteristics and physical injury. The reason for this connection is not clear. As suggested above, it could be the case of the lack of social support, and heavy mental strains lead healthcare staff to engage in unsafe work practices. It could also be the case that in work 
environments that are not supportive the leadership ethics does not concern itself enough with safety protocols to ensure compliance with its staff. The last explanation seems to be a likely candidate, a discussion the researcher will turn to shortly. The consequences of a poor work environment appear to increase healthcare training and healthcare training in staff. What are the related consequences of a poor work environment that can perhaps lead to a higher than average rate of injury? One answer offered by the literature is the underreporting of injuries. A study of 28 units in 20 hospitals in the Netherlands examined the relationship between 11 workplace cultural dimensions and six types of unintended events, which were collected through staff reporting. Even though most of the dimensions did not show a relationship with those unintended events, three of those dimensions were significantly related; non-punitive response to error, hospital management support, and willingness to report (Smits, Wagner, Spreewenberg, Timmermans, Wal \& Growenewegen, 2012).

The last dimension was the most significantly related, which is telling. This research combined with the above studies suggests part of the reason cultural dimensions' effect injury and healthcare training have to do with the comfort of the staff in reporting those incidences. If the leadership ethics appears to be unresponsive (nonpunitive response to error), then it can discourage staff from reporting. This could, in turn, give the workplace the impression that safety is not an issue worth investing in, and the staff has the impression it is not a high priority. This appears to be a large issue in private hospitals where Menzel (2008) shows that the US Department of Labor and the Bureau of Labor Statistics underreport the incidence of injury. The author argues that this is, in part a result of poor reporting procedures within the hospital environment. Whether through accident or design these procedures either encourage or discourage reporting. The author argued that improved reporting procedures are necessary to reflect the rate of injury accurately. This piece of information regarding injury reporting is particularly troublesome, given that the rate of reported injury is already so high. The ANA Health and Safety Survey (2011) confirmed this information and was conducted in 2001 as well, which allows us to see how this may have changed in 10 years. The results are not promising. While this number is not available in the 2011 survey, some other related information was collected. For example, 80\% of the 4,614 respondents reported frequently working despite the musculoskeletal pain. This compliments the fact that they found only $10 \%$ reported missing any work due to injury in 12 months, even though $42 \%$ reported being injured in that same 12-month period, and 56\% reported experiencing musculoskeletal pain caused or made worse by their job. These results suggest that there is a disconnect between the scientific evidence for a major public health problem and the actual implementation of measures to address the problem.

Thus far, the research suggests that workplace characteristics can have a strong impact on the prevalence of healthcare training among healthcare workers and that work-related support measures seem to influence the rate of injury. Earlier the researcher cited a study that showed that perceived lack of support from immediate supervisor and lack of pleasant and relaxing culture at work were associated with LBP-related work leaves. Previously the researcher also postulated that this could be because, in poor work environments, safety procedures are not emphasized and, therefore, not always a priority by the staff or leadership ethics. However, this study regarding presenteeism complicates that assessment slightly. The relationship between workplace culture and injury appears more complex than one might think. So, what specific behaviors contribute to increased healthcare training in workplaces with low levels of safety culture? One clue may be that nurses are less likely to employ safety protocols such as not using healthcare training equipment in work environments that do not emphasize safety. One common complaint among staff is that using equipment takes too long; this is also supported by the ANA survey which showed that even though the equipment is more available, it is not employed $100 \%$ of the time it is called for (ANA Survey, 2011; Kanaskie \& Snyder, 2018; Mechan, 2014). Researchers challenged this assumption by timing how long it takes to use a variety of lift equipment to perform a variety of routine procedures. The longest duration was 3 minutes, 41 seconds, which challenges the notion that these procedures take up too much time (Mechan, 2014).

It could also be the case the certain workplace norms that are not explicitly related to safety could affect safety in the workplace. Myers, Shoenfishch, \& Lipscomb (2012) examined the relationship between workplace culture and the employment of certain safety protocols in two acute care facilities. The authors differentiated between "safety culture" and workplace culture to determine if other types of social norms impact safety practices. They used interviews with focus groups to collect date from nurse staff $(n=39)$ and physical therapy and occupational therapy staff $(n=17)$. They found that most respondents had a patient-centered approach to patient care, but that the implied purpose of lifting equipment clashes with nurse's cultural emphasis on compassion, and physical therapists' emphasis on independence, except where lifting equipment increases independence. This implies that nurses and physical therapists may not always employ the use of safe patient equipment if they feel it undermines compassion or independence. This may be a particular problem in geriatric 
centers where the use of lifts to move patients may impact their psychological well-being. Unfortunately, these patients' size makes them even a greater risk to the direct care worker.

Another cultural consideration that may be worth exploring is generational differences. Gallagher (2012) argues that these differences should be considered when implementing a healthcare training program because different generations respond to different kinds of training in different ways. The author argues that for the oldest generation, the "Silent Generation," a more top-down, structured work environment is what they are accustomed. This generation is the oldest, they would be in their 70s or so now, so most have retired, and the "Baby Boomers" now hold $82 \%$ of leadership ethics positions in healthcare. The author argues that this generation is much more team and consensus-oriented and that training for these individuals should focus on making them more comfortable with technology. This is particularly crucial when considering that $62 \%$ of the 4,614 nurses surveyed by the ANA in 2011 were over 50 (ANA, 2011). This information also lends support to the current study. If most nurses are over 50, then most (or all of them) were trained to use manual handling techniques rather than the evidence-based techniques that are effective. This provides even more evidence that a training program, administered at certain intervals can reduce the rate of injury and its associated costs.

The author goes on to argue that the younger generation, generation $\mathrm{x}$, is much more individualist and desires immediate feedback on performance. They are much blunter and less team-oriented than the older generation. These considerations could play a role in determining the type and degree of training. Gen Xers are much more comfortable with technology, so perhaps if the given facility has a younger staff, the use of equipment might be a smoother transition than with older generations. On the flip side, if a facility has an older workforce, it may require more effort to train them to use technology. Research has shown that cultural considerations play a complex role in the implementation of Healthcare training programs and protocols. The specific characteristics of the work facility, the type of caregiver that is employed, preexisting cultural norms, leadership ethics, and age distribution all play an integral role in determining the success of a healthcare training program. This means that programs may be tailored to each facility to produce and sustain and strong safety culture in the workplace. Next, the researcher will turn to a case study of a program implementation that successfully changed the safety culture of the facility.

\section{Conclusion}

The researcher has established that the healthcare industry has a susceptibility to healthcare training compared with most other industries (Gomaa et al., 2015; Nelson, 2006; NIOSH, 2016; U.S. Department of Labor, 2017). Also, they have one of the highest overexertion rates of any other profession, with a rate of 76 per 10,000 workers. This is not simply a national problem; it is present in hospitals across the world (Berthelette D., Leduc N., Bilodeau H., Durand M. J. \& Faye C., 2012). The consequences of this are both a massive human cost and massive monetary cost, to the tune of about $\$ 20$ Billion annually (O'Connell, 2013). One of the causes of this issue is the increase in workloads, both due to higher bed capacities and increased incidence of obesity in patients (Gomaa et al., 2015; Lipley, 2012; Sang \& Brings, 2015). These two factors may help explain why this problem persists over time, despite efforts by many groups to raise awareness (American Nurses Association, 2013). While 11 states have passed legislation, most of the laws apply only to some care facilities and no other, and most do not mandate specific kinds of interventions that have been shown to reduce the rate of injuries and their associated costs (Lee et al., 2015; Powell-Cope \& Rugs, 2015). Given that the rate of injury has not declined on a national scale, it is imperative that steps be taken at the national level to ensure compliance across the country. It must be a priority to emphasize that these programs save money (even though there is an initial outlay), studies have shown that costs can be recouped quickly if programs are implemented correctly (Alamgir et al., 2015).

It is obvious from the literature that care facilities have attempted a variety of approaches to try to combat these issues, including the use of healthcare training, ergonomic equipment, education, and training with varying degrees of success. It seems that most studies that use a baseline comparison type of approach find that most, if not all, of these protocols are effective at reducing injuries. However, Burdorf, Kopplaar, \& Evanoff (2013) found that the effect of equipment was minimal when multiple studies were taken into consideration. It is possible that most studies focus on programs that have been recently or more vehemently implemented. This may exaggerate the more commonplace use of equipment in those facilities where it is strongly emphasized. This lends potential support to the evidence regarding cultural considerations. That is, for Healthcare training programs to produce the desired reduction in injuries, the programs must be very well implemented from the top-down and bottom-up. It is clear from the case study described by Goetz \& TaylorTrujillo (2012) that a program must employ a variety of components. Also, a good deal of research is necessary to determine how the program can be most effectively implemented. This last discussion lends a good deal of support for the researcher's choice to use the Kirkpatrick Model to evaluate training methods. The researcher 
would like to note the common aspects of the model with the healthcare training recommendations for a welldesigned healthcare training protocol. Both models emphasize feedback from participants (reaction), training and assessment (learning), focus on modifying behaviors, and outcomes (results, or the degree to which the program reduces injuries). These are also emphasized by the case study described above. The researcher intends to investigate how often Healthcare training must be repeated to maintain reductions in injuries to healthcare staff. Many programs employ retraining, but no research shows how often this retraining must occur in Healthcare training programs to maximize results by decreasing staff injuries and minimize associated costs. For facilities that engage in monthly or bi-monthly training, they may be doing more than is necessary. Those facilities that engage in only annual training may similarly be contributing to increased costs to their facilities. This is the subject of this research.

Author Contributions. Conceptualization, Dr. Kasztelnik \& Dr. Probst; data curation, Dr. Kasztelnik \& Dr. Probst; formal analysis, Dr. Kasztelnik \& Dr. Probst; investigation, Dr. Kasztelnik \& Dr. Probst; methodology, Dr. Kasztelnik \& Dr. Probst; project administration, Dr. Kasztelnik; resources, Dr. Kasztelnik \& Dr. Probst; software, Dr. Kasztelnik \& Dr. Probst; supervision, Dr. Kasztelnik; validation, Dr. Kasztelnik; visualization, Dr. Kasztelnik \& Dr. Probst; writing - original draft, Dr. Kasztelnik \& Dr. Probst; writing - review \& editing, Dr. Kasztelnik \& Dr. Probst.

Funding. There is no funding for this research.

\section{References}

1. Abdulghani, H.M., Shaik, S.A., Khamis, N., Al-Drees, A.A., Irshad, M., Khalil, M., Alhaqwi, A.I., \& Isnani, A. (2014). Research methodology workshops evaluation using the Kirkpatrick's Model: Translating theory into practice. Medical Teacher, 36, 24-29. doi:10.3109/0142159X.2014.886012.

2. Al-Sabah, S., Al Haddad, E., \& Khwaja, H. (2018). The prevalence of musculoskeletal Injuries in Bariatric Surgeons. Bariatric Times, 15(3), 16-19. Available at: https://bariatrictimes.com/musculoskeletal-injuriesbariatric-surgeons-march-2018/.

3. Amin, N.A., Fatt Quek, K., Oxley, J.A., Noah, R., \& Nordin, R. (2018). Emotional distress as a predictor of work-related musculoskeletal disorders in Malaysian nursing professionals. International Journal of Occupational \& Environmental Medicine, 9(2), 69-78. doi:10.15171/ijoem.2018.1158.

4. Arnold, M., Wilson, C., McIlvaince, J., Labreche, M., \& Stevens, L. (2015). Integrating mobility and safe patient handling. American Journal of Safe Patient Handling, 5(2), 1-20. Available at: https://sphmjournal.com/product/june2015v5n2-mobility-supplement-2/.

5. Arnold, M., Combs, J., Gach, R., \& Labreche, M. (2015). Overcoming barriers to mobilizing bariatric patients: Three case studies. American Journal of Safe Patient Handling, 5(2), 47-54. Available at: https://sphmjournal.com/product/june2015v5n2-overcoming-barriers/.

6. Babbie, E. R. (2012). The practice of social research. Belmont, CA: Wadsworth. Available at: http://ccftp.scu.edu.cn/Download/e6e50387-38f2-4309-af84-f4ceeefa5baa.pdf.

7. Baker, D., Pynsent, P. B., Fairbank, J. T., Davidson, M., \& Keating, J. L. (2002). Oswestry Low Back Pain Disability Questionnaire - revised. Physical Therapy, 82, 8-24. https://doi.org/10.1093/ptj/82.1.8.

8. Berthelette, D., Leduc, N., Bilodeau, H., Durand, M.J., \& Faye, C. (2012). Evaluation of the implementation fidelity of an ergonomic training program designed to prevent back pain. Applied Ergonomics, 43, 239-245. https://doi.org/10.1016/j.apergo.2011.05.008.

9. Bryman, A. (2012). Social research methods (4th ed.). Oxford, England: Oxford University Press. Available at: https://www.academia.edu/30520568/Social_Research_Methods_4th_Edition_by_Alan_Bryman_pdf.

10.Burdorf, A., Koppelaar, E., \& Evanoff, B. (2013). Assessment of the impact of lifting device use on low back pain and musculoskeletal injury claims among nurses. Occupational and Environmental Medicine, 70(7), 491. doi: 10.1136/oemed-2012-101210.

11.Celona, J. (2014). Elements of a successful safe patient handling and mobility program. American Nurse Today, 9(9), 4-6. Available at: https://www.myamericannurse.com/wp-content/uploads/2014/09/ant9Patient-Handling-Supplement-821a Elements.pdf.

12.Chiwaridzo, M., Makotore, V., Dambi, J. M., Munambah, N., \& Mhlanga, M. (2018). Work-related musculoskeletal disorders among registered general nurses: a case of a large central hospital in Harare, Zimbabwe. BMC Research Notes, 11(1), 315. doi:10.1186/s13104-018-3412-8.

13.Clarke, R. (2017). Healthcare worker decompression sickness: incidence, risk, and mitigation. Undersea \& Hyperbaric Medicine: Journal of The Undersea and Hyperbaric Medical Society, Inc, 44(6), 509-519. DOI:10.22462/11.12.2017.2. 
14.Coenen, P., Douwes, M., van den Heuvel, S., \& Bosch, T. (2016). Towards exposure limits for working postures and musculoskeletal symptoms-a prospective cohort study. Ergonomics, 59(9), 1182-1192. doi:10.1080/00140139.2015.1130862.

15.Dabholkar, T., Yardi, S., Dabholkar, Y., Velankar, H., \& Ghuge, G. (2017). A survey of work-related musculoskeletal disorders among otolaryngologists. Indian Journal of Otolaryngology \& Head \& Neck Surgery, 69(2), 230-238. doi:10.1007/s12070-017-1106-5.

16.Darragh, A., \& Campo, M. (2017). Health-related quality of life associated with severity and age in occupational and physical therapists with musculoskeletal disorders. International Journal of Safe Patient

Handling \& Mobility (HEALTHCARE TRAINING), 7(1), 29-39. Available at: https://sphmjournal.com/product/health-related-quality-of-life-associated-with-severity-and-age-inoccupational-and-physical-therapists-with-musculoskeletal-disorders/.

17.Darragh, A. R., Mariya, S., Margulis, H., \& Campo, M. (2014). Effects of a safe patient handling and mobility program on patient self-care outcomes. American Journal of Occupational Therapy, 68(5), 589596. doi:10.5014/ajot.2014.011205.

18.Edwards, J., Davey, J., \& Armstrong, K. (2013). Returning to the roots of safety culture: A review and reconceptualization of safety culture. Safety Science, 55, 70-80. doi: 10.1016/j.ssci.2013.01.004.

19.Elnitsky, C. A., Lind, J. D., Rugs, D., \& Powell-Cope, G. (2014). Implications for patient safety in the use of safe patient handling equipment: A national survey. International Journal of Nursing Studies, 51(12), 1624-1633. doi: 10.1016/j.ijnurstu.2014.04.015.

20.Enos, L. (2013). Safe patient handling and patient safety: Identifying the current evidence base and gaps in research. American Journal of Safe Patient Handling \& Movement, 3(3), 94-102. Available at: https://sphmjournal.com/product/september-2013-v3n3-sph-and-patient-safety-identifying-the-currentevidence/.

21.Epstein, S., Sparer., Tran, B., Ruan, Q., Singhal, D., Lee, B., \& Dennerlein, J. (2018). Prevalence of workrelated musculoskeletal disorders among surgeons and interventionalists: A systematic review and metaanalysis. JAMA Surgery, 153(2). doi:10.1001/jamasurg.2017.4947.

22.Eriksen, W., Bruusgaard, D., \& Knardahl, S. (2004). Work factors as predictors of intense or disabling low back pain; a prospective study of nurses' aides. Occupational and Environmental Medicine, 61(5), 398-404. http://doi.org/10.1136/oem.2003.008482.

23.Fairbank, J.C.T., Couper, J., \& Davies, J.B. (1980). The Oswestry Low Back Pain Questionnaire. Physiotherapy, 66, 271-273. Available at: https://aaos.org/globalassets/quality-and-practiceresources/patient-reported-outcome-measures/spine/oswestry-2.pdf.

24.Fairbank, J.C.T., \& Pynsent, P.B. (2000). The Oswestry Disability Index. Spine, 25(22), 2940-2953. DOI: $10.1097 / 00007632-200011150-00017$.

25.Gallagher, S.M. (2012). Intergenerational considerations in sustaining safe patient handling and mobility success: Implications in equipment usage. American Journal of Safe Patient Handling \& Movement, 2(4), 150-137. Available at: https://sphmjournal.com/product/december-2012-intergenerational-considerations/.

26.Gallagher, S. (2015). A practical guide to bariatric safe patient handling. American Journal of Safe Patient Handling, 5(2), 72. Available at: https://sphmjournal.com/product/june2015v5n2-book-review/.

27.Goetz, S. B., \& Taylor-Trujillo, A. (2012). A change in culture: Violence prevention in acute behavioral health setting. Journal of American Psychiatric Nurses Association, 18(2), 96-103. DOI: $10.1177 / 1078390312439469$.

28.Gomaa, A. E., Tapp, L. C., Luckhaupt, S. E., Vanoli, K., Sarmiento, R. F., Raudabaugh, W. M., \& Sprigg, S. M. (2015). Occupational traumatic injuries among workers in healthcare facilities - United States, 20122014. Mmwr-Morbidity and Mortality Weekly Report, 64(15), 405-410. Available at: https://www.cdc.gov/mmwr/preview/mmwrhtml/mm6415a2.htm.

29.Harwood, K., Scalzitti, D.A., Campo, M., \& Darragh, A.R. (2016). A systematic review of safe patient handling and mobility programs to improve patient outcomes in rehabilitation. American Journal of Safe Patient Handling and Mobility, 6(4), 141-150. Available at: https://hsrc.himmelfarb.gwu.edu/smhs crl facpubs/155/.

30.Howard, N. (2016). Special feature: Safe patient handling \& mobility programs and policies: short term vs. long term evaluation. American Journal of Safe Patient Handling and Mobility, 6(3), 130-131. Available at: https://sphmjournal.com/product/september2016v6n3-special-feature-sphm-programs-and-policies/.

31.Hsin-Yi Kathy Cheng, Man-Ting Wong, Yu-Chung Yu, \& Yan-Ying Ju. (2016). Work-related musculoskeletal disorders and ergonomic risk factors in special education teachers and teacher's aides. BMC Public Health, 16(1), 1-9. https://doi-org.lopes.idm.oclc.org/10.1186/s12889-016-2777-7. 
32.Kirkpatrick, D. L. (1998). Evaluating training programs: The four levels. Oakland, CA: Berrett-Koehler Publishers. Available at:

https://www.academia.edu/3183613/Evaluating Training Programs The Four Levels.

33.Lipley, N. (2012). Safety compromised by rising workloads. Nursing Management - UK, 19(5), 5. DOI:10.7748/nm2012.09.19.5.5.p9257.

34.Mayeda-Letourneau, J. (2014). Safe patient handling and movement: A literature review. Rehabilitation Nursing, 39(3), 123-129. doi:10.1002/rnj.133.

35.Mechan, P. (2014). Challenging the myth that it takes too long to use safe patient handling and mobility technology: A task time investigation. American Journal of Safe Patient Handling \& Movement, 4(2), 46-51. Available at: https://sphmjournal.com/product/challenging-the-myth-that-it-takes-too-long-to-use-sphm/.

36.Menzel, N. (2008). Underreporting of musculoskeletal disorders among healthcare workers: research needs. AAOHN Journal, 56(12), 487-494. doi:10.3928/08910162-20081201-06.

37.Menzel, Feng, \& Doolen (2016). Low back pain in student nurses: Literature review and prospective cohort study. International Journal of Nursing Education Scholarship, 13(1), 1-21. Available at: https://digitalscholarship.unlv.edu/nursing_fac_articles/171/.

38.Monaghan, H.M. (2013). Safe patient handling \& movement: Legislation, accreditation, quality, and recognition. American Journal of Safe Patient Handling \& Movement, 3(3), 103-109. Available at: https://sphmjournal.com/product/september-2013-v3n3-sphm-legislation-accreditation-quality-andrecognition/.

39.Myers, D. J., Schoenfisch, A. L., \& Lipscomb, H. J. (2012). Cultural influences on workplace safety: An example of hospital workers' adoption of patient lifting devices. Safety Science, 50(3), 494-501. doi:http://dx.doi.org.library.gcu.edu:2048/10.1016/j.ssci.2011.10.015.

40.Nelson, A. (Ed). (2006). Safe patient handling and movement, a practical guide for healthcare professionals. New York, NY: Springer Publishing Company. Available at: https://www.researchgate.net/publication/232214653 Safe Patient Handling and Movement Preventin g_back_injury_among_nurses_requires_careful_selection_of_the_safest_equipment_and_techniques_The _second_of_two_articles.

41.Oakman, J., Rothmore, P., \& Tappin, D. (2016). Intervention development to reduce musculoskeletal disorders: Is the process on target? Applied Ergonomics, 56, 179-186.doi: 10.1016/j.apergo.2016.03.019.

42.Occupational Safety and Health Administration (2013). Integrating patient and workplace safety programs (OSHA 3730). 2013. Available at https://wwwlosha.gov/Publications/OSHA3730.pdf, Accessed date: 26 June 2018.

43.O'Connell, D. (2013). Focus on safe lifting protects nurses, patients. Alaska Nurse, 63(1), 1-6. Available at: http://www.aknurse.org/layouts/aknurse/files/documents/misc/ak3 13.pdf.

44.Olson, R., Thompson, S. V., Elliot, D. L., Hess, J. A., Rhoten, K. L., Parker, K. N., \& Marino, M. (2016). Safety and health support for home care workers: The COMPASS randomized controlled trial. American Journal of Public Health, 106(10), 1823-1832. doi:10.2105/AJPH.2016.303327.

45.Perez, A. (2016). An evidence-based approach to safe patient handling and mobility education. American Journal of Safe Patient Handling \& Movement, 6(3), 113-119. Available at: https://sphmjournal.com/product/september2016v6n3-an-evidence-based-approach-to-sphm-education/.

46.Peterson, M. J., Kahn, J. A., Kerrigan, M. V., Gutmann, J. M., \& Harrow, J. J. (2015). Pressure ulcer risk of patient handling sling use. Journal of Rehabilitation Research \& Development, 52(3) 291-300. doi:10.1682/JRRD.2014.07.0172.

47.Powell-Cope, G., \& Rugs, D. (2015). What elements of the 2013 American Nurses Association safe patient handling and mobility standards are reflected in state legislation? American Journal of Safe Patient Handling and Mobility, 5(1), 13-18. Available at: https://www.ncbi.nlm.nih.gov/pmc/articles/PMC4581891/.

48.Powell-Cope, G., Toyinbo, P., Patel, N., Rugs, D., Elnitsky, C., Hahm, B., \& Hodgson, M. (2014). Effects of a national safe patient handling program on nursing injury incidence rates. The Journal of Nursing Administration, 44(10), 525-534. DOI:10.1097/NNA.0000000000000111.

49.Purnomo, H., Giyono, E., \& Apsari, A. (2017). The use of macro-ergonomic work system designs to reduce musculoskeletal disorders and injury risk in training. South African Journal of Industrial Engineering, 1, 47. doi:10.7166/28-1-1600.

50.Schoenfishch, A.L., Lipscomb, H.J., Myers, D.J., Fricklas, E., \& James, T. (2011). A lift assist team in an acute care hospital-prevention of injury of transfer of risk during patient handling tasks? Journal of the American Association of Occupational Health Nurses, 59(8), 329-334. doi: 10.3928/08910162-20110726-02. 
51.Shameela, T.V., Veena Pais, Shaikhji Saad \& Nusaibath M. (2015). Correlation of low back pain with body mass index, functional reach test among female nursing professionals. International Journal of Physiotherapy, 2(6), 894-898. DOI: https://doi.org/10.15621/ijphy/2015/v2i6/80745.

52.Singh B., \& Paudel B. (2015). Knowledge and practice of healthcare workers regarding needle stick injuries in a tertiary care center of Nepal. Kathmandu University Medical Journal, 51(3), 230-233. DOI:https://doi.org/10.3126/kumj.v13i3.16813.

53.Smits, M., Wagner, C., Spreewenberg, P., Timmermans, D.R., Wal, G., \& Growenewegen, P.P. (2012). The role of patient safety culture in the causation of unintended events in hospitals. Journal of Clinical Nursing, 21, 3392-3401. DOI:10.1111/j.1365-2702.2012.04261.x.

54.Stevenson, J., Hecksel, K., Deneau, K., \& Dudley, B. (2011). Do student physical therapists learn and practice safe patient handling in entry-level physical therapy education programs? American Journal of Safe Patient Handling \& Movement, 1(4), 8-16. Available at: https://sphmjournal.com/product/december2011-v1n4-do-student-physical-therapists-learn-and-practice-safe-patient-handling-in-entry-levelphysical-therapy-education-programs/.

55.Strong, J., Ashton, R., \& Stewart, A. (1994). Chronic low back pain: Toward an integrated psychosocial assessment model. Journal of Consulting and Clinical Psychology, 62(5), 1058-1063. doi:10.1037/0022006X.62.5.1058.

56.Taderera, B. H., Heinrich Hendricks, S. J., Pillay, Y., \& Hendricks, S.H. (2017). Human resource for health reform in peri-urban areas: a cross-sectional study of the impact of policy interventions on healthcare workers in Epworth, Zimbabwe. Human Resources for Health, 15(1), 1-13. doi:10.1186/s12960-017-0260-x.

57.Thomas, D.R., \& Thomas, Y.N. (2014). Review: Interventions to reduce injuries when transferring patients: A critical appraisal of reviews and a realist synthesis. International Journal of Nursing Studies, 51, 1381-1394. doi: 10.1016/j.ijnurstu.2014.03.007.

58.van der Beek, A.J., Dennerlein, J.T. (Jack T.), Huysmans, M.A. (Maaike A.), Mathiassen, S.E, Burdorf, A., van Mechelen, W., Coenen, P. (2017). A research framework for the development and implementation of interventions preventing work-related musculoskeletal disorders. Scandinavian Journal of Work, Environment and Health, 43(6), 526-539. doi:10.5271/sjweh.3671.

59.Vieira, E., Schneider, P., Guidera, C., Gadotti, I., \& Brunt, D. (2016). Work-related musculoskeletal disorders among physical therapists: A systematic review. Journal of Back and Musculoskeletal Rehabilitation, 29(3), 417-428. doi:10.3233/BMR-150649.

60.Vollman, K. M., \& Bassett, R. (2014). Transforming the culture: The key to hardwiring early mobility and safe patient handling. American Nurse Today, 7-25. Available at: https://www.myamericannurse.com/transforming-culture-key-hardwiring-early-mobility-safe-patienthandling/.

61.Waters, T.R. (2007). When is it safe to manually lift a patient? American Journal of Nursing, 107(8), 53-58. DOI: 10.1097/01.NAJ.0000282296.18688.b1.

62.Waters, T., Collins, J., Galinsky, T., \& Caruso, C. (2006). NIOSH research efforts to prevent musculoskeletal disorders in the healthcare industry. Orthopaedic Nursing 25(6), 380-389. DOI:10.1097/00006416-200611000-00007.

63.Wanless, S. (2017). Applying theories of health behavior and change to moving and handling practice. International Journal of Safe Patient Handling \& Mobility (HEALTHCARE TRAINING), 7(3), 105-109. Available at: https://sphmjournal.com/product/applying-theories-health-behavior-change-movinghandling-practice/.

64.Williams, C., Haines, T., Penkala, S., Smith, P., \& Bowles, K. (2017). Exploring musculoskeletal injuries in the podiatry profession: An international cross-sectional study. Journal of Foot and Ankle Research, 10(1). doi:10.1186/s13047-016-0185-y.

65.Wilson, C., Beaumont, M., Alberstadt, K., Drake, J.M., Ednalino, K.G., Thornsberry, M.M., \& Zahringer, S.G. (2011). The effectiveness of a patient handling education program for Nursing Assistants. Journal of Acute Care Physical Therapy, 2(1), 12-38. Available at: https://cdn.ymaws.com/www.acutept.org/resource/resmgr/imported/JACPT\%202011-1.pdf.

66. Yoshikawa, H., Weisner, T., Kalil, A., \& Way, N. (2013). Mixing qualitative and quantitative research in developmental science: Uses and methodological choices. Developmental Psychology, 44(2), 344-354. doi:10.1037/0012-1649.44.2.344.

67.Zadvinskis, I.M., Wills, C.E., Chipps, E., \& Patterson, E.S. (2013). Process measurement in safe patient handling and movement: A literature review. American Journal of Safe Patient Handling \& Movement, 3(2), 55-63. Available at: https://sphmjournal.com/product/june-2013-process-measurements-in-sphm-aliterature-review/. 